\title{
Evaluation of the risk factors of depressive disorders comorbid with obstructive sleep apnea
}

This article was published in the following Dove Press journal:

Neuropsychiatric Disease and Treatment

16 January 2017

Number of times this article has been viewed

\section{Liqiang Cai' \\ Luoyi $\mathrm{Xu}^{\prime}$ \\ Lili Wei' \\ Yi Sun ${ }^{2}$ \\ Wei Chen ${ }^{1,3}$}

'Department of Psychiatry, Sir Run Run Shaw Hospital, Collaborative Innovation Center for Brain Science, Zhejiang University School of Medicine, ${ }^{2}$ Department of Electroencephalogram, Sir Run Run Shaw Hospital, ${ }^{3}$ Key Laboratory of Medical Neurobiology, Chinese Ministry of Health, Zhejiang University School of Medicine, Hangzhou, Zhejiang, People's

Republic of China
Correspondence: Wei Chen

Department of Psychiatry, Sir Run Run Shaw Hospital, Collaborative Innovation Center for Brain Science, Zhejiang University School of Medicine, 3 East Qingchun Road, Hangzhou 310016, Zhejiang, People's Republic of China Tel +86 57l 8788 780 I

Email srrcw@zju.edu.cn
Objective: Overlap of obstructive sleep apnea (OSA) complicates diagnosis of depressive disorder and renders antidepressant treatment challenging. Previous studies have reported that the incidence of OSA is higher in patients with depression than in the general population. The purpose of this article was to investigate clinical risk factors to predict OSA in depression disorders.

Methods: A total of 115 patients diagnosed with major depressive disorder (MDD) and bipolar disorder (in a major depressive episode), who underwent overnight polysomnography, were studied retrospectively. They were divided into two groups: non-OSA and OSA. The patients who had apnea-hypopnea index (AHI) $<5$ were defined as the non-OSA group, whereas the OSA group was defined as those with an $\mathrm{AHI} \geq 5$. Logistic regression was used to analyze the association among AHI and clinical factors, including sex, age, body mass index (BMI), Hamilton Depression Rating Scale (HAMD), Hamilton Anxiety Rating Scale, Pittsburgh Sleep Quality Index (PSQI), and diagnosis (MDD or bipolar disorder [in a major depressive episode]).

Results: In 115 patients, 51.3\% had OSA. Logistic regression analysis showed significant associations between AHI and diagnosis (MDD or bipolar disorder [in a major depressive episode]), BMI, HAMD, and PSQI $(P<0.05)$.

Conclusion: The findings of our study suggested that the rate of depression being comorbid with OSA is remarkably high and revealed that there is a high rate of undetected OSA among depressive disorder patients and untreated OSA among mood disorder patients. The clinical risk factors (diagnosis [MDD or bipolar disorder \{in a major depressive episode\}], BMI, HAMD, and PSQI) could predict AHI or OSA diagnosis and contribute to OSA screening in depressive disorder patients.

Keywords: major depressive disorder, polysomnography, obstructive sleep apnea, risk factors, apnea-hypopnea index

\section{Introduction}

Depression is one of the major causes of ill health and economic burden worldwide. Antidepressant medication is a standard treatment and the first choice for depressed patients in current psychiatric guidelines. ${ }^{1}$ Approximately $60 \%$ of patients respond well to active treatment, ${ }^{2-4}$ but $\sim 40 \%-50 \%$ of patients using the full dosage and duration of antidepressant medications still have residual symptoms and cannot obtain full remission. ${ }^{4-6}$ One reason for this may be that some depressed patients discontinue treatment because of adverse effects or higher economic burden. ${ }^{2}$ Moreover, misdiagnosis, genetic inheritance, and comorbid somatic disease also influence the outcome of treatment. ${ }^{7,8}$ In a 5-year follow-up study, Riihimäki et al ${ }^{9}$ studied 137 major depressive disorders (MDDs) and found that the patients spent $34 \%$ of the follow-up time 
in major depressive episodes, $24 \%$ in partial remission, and $42 \%$ in full remission.

Szaulińska et $\mathrm{al}^{10}$ reported that obstructive sleep apnea (OSA) is present in $11 \%-18 \%$ of patients with MDDs, $15 \%-48 \%$ of patients with schizophrenia, and $21 \%-43 \%$ of patients with bipolar disorder, but in only $5 \%$ of the general population. ${ }^{11}$ One study has shown that patients diagnosed with OSA are at an increased risk of a diagnosis of depression. ${ }^{12}$ Additionally, the symptoms of sleep apnea might imitate the symptoms of depression, including sleep disturbance, general fatigue, decreased volition and judgment ability, aggravated cognitive impairment, and a lower quality of life. ${ }^{10}$ In addition, OSA might exacerbate symptom severity in MDDs. ${ }^{8}$ Participants with both MDDs and OSA also reported more severe and longer episodes of depression. ${ }^{13}$ Meanwhile, OSA might influence response to pharmacological treatment of depression as well as reduce patient adherence to antidepressants. ${ }^{8,14}$ Waterman et al ${ }^{14}$ studied 400 patients with MDDs and found that those with comorbid OSA were 1.5 times less likely to respond to 12 weeks of treatment with the antidepressant venlafaxine than those with either depressive disorder occurring alone. Kerner et al ${ }^{15}$ confirmed that the cognitive performance of MDDs comorbid with severe OSA is even worse than that of mild to moderate OSA, while some reports suggest that depressive symptoms might be improved in at least some patients receiving continuous positive airway pressure therapy or other treatment for OSA. ${ }^{16-19}$ From the point of view of the physicians, this suggests that screening and treatment for OSA is very important. ${ }^{10}$

In depressive disorders, comorbid OSA is even higher. ${ }^{10}$ Physicians should consider screening for OSA when assessing patients for depression, as it may indicate more difficulty to treat depression. ${ }^{8}{ }^{819}$ However, it is difficult to define who actually is at risk of OSA in depressive disorders. This has prompted the need to identify OSA using appropriate risk factors in clinical settings. Multiple studies have found that sex, age, obesity, snoring, pharyngeal abnormalities, and cephalometric features are risk factors for OSA in the general population. ${ }^{20-22}$ Hattori et al $^{23}$ studied 32 mood disorder patients and found that appropriate OSA risk factors (such as apnea, snoring, and body mass index [BMI]) may help to better identify OSA. ${ }^{23}$ Nonetheless, to our knowledge, there have been few studies on risk factors for OSA in depressive disorders. We screened OSA patients with mood disorders presenting with depression using polysomnography (PSG) and examined which or how many risk factors would contribute most to OSA screening.

\section{Methods}

\section{Participants}

All participants provided written informed consent to be included in the study, and the study design was approved by the ethics committee of Sir Run Run Shaw Hospital, Zhejiang University School of Medicine. We reviewed 1,091 medical files of patients who had undergone in-room PSG from December 2013 to December 2015. The inclusion criteria were as follows: 1) diagnosis of an MDD or a bipolar disorder (in a major depressive episode) through a structured MiniInternational Neuropsychiatric Interview (MINI) based on the Diagnostic and Statistical Manual of Mental Disorders, fourth edition (DSM-IV);2) patients aged 18 years or older; and 3 ) the Hamilton Depression Rating Scale (HAMD; 17 items) $\geq 10$. Patients with $\mathrm{BMI} \geq 30 \mathrm{~kg} / \mathrm{m}^{2}$, underlying respiratory disease, history of taking drugs that can affect normal sleep architecture and psychotic symptoms, suicidal thoughts, restless legs syndrome, diagnosis of other sleep-related disorder, other clinical phases of bipolar disorder, and a previous history of treatment for OSA were excluded. Patients with mood disorder who had undergone no pharmacological therapy were also excluded. A total of 115 patients met the criteria and were divided into non-OSA $(\mathrm{n}=56)$ and OSA $(\mathrm{n}=59)$ groups according to PSG results. Patients who had apnea-hypopnea index (AHI) $<5$ were defined as the non-OSA group, whereas the OSA group was defined as those with an AHI $\geq 5$.

\section{Polysomnography}

PSG recording was performed using Trackit 32/0 (Lifelines Ltd, Hants, UK). Electroencephalography, electrocardiogram, and electromyography of chin and leg; arterial oxygen saturation $\left(\mathrm{SaO}_{2}\right)$; airflow at nose and mouth; chest and abdominal movement; and body position were recorded. Measurements were made from 21.00 hours to 06.00 hours the following morning in a quiet room. Apnea events were defined as complete cessation or near-complete cessation of airflow for $\geq 10$ seconds, and hypopnea events were based on a $30 \%$ reduction of airflow for 10 seconds accompanied by $\mathrm{SaO}_{2}$ reduction of $>3 \%$. The number of episodes of apnea and hypopnea per hour during total sleep time was calculated, and the result was the AHI.

\section{Assessment}

The HAMD, the Hamilton Anxiety Rating Scale (HAMA), and the Pittsburgh Sleep Quality Index (PSQI) were used for included patients when they underwent PSG.

\section{Statistical analysis}

To evaluate the relation between clinical factors (sex, age, and BMI, HAMD, HAMA, PSQI, diagnosis [MDD or 
bipolar disorder]), and AHI, logistic regression was used. All statistical analysis was performed using SPSS 16.0 (SPSS Inc., Chicago, IL, USA), and $P<0.05$ was considered as statistically significant.

\section{Results}

The mean age of the patients was $51.58 \pm 11.48$ years. Seventyfour patients were diagnosed with MDD, and 41 were diagnosed with bipolar affective disorder. Their mean HAMD score was $20.97 \pm 4.47$ for 17 items. On PSG, 59 patients were diagnosed with OSA $(\mathrm{AHI} \geq 5)$ and 56 patients were diagnosed with non-OSA (AHI $<5$; Table 1).

When referring to the correlation between risk factors and AHI, the results showed a significant association between the AHI and diagnosis (MDD or bipolar disorder [in a major depressive episode]), BMI, HAMD, and PSQI (Table 2).

\section{Discussion}

In all, 15\%-30\% of patients with depressive disorder were classified as having treatment-resistant depression. ${ }^{23}$ A previous study reported that OSA is present in $11 \%-18 \%$ of patients with MDDs. ${ }^{10}$ From this, it is reasonable to assume that some treatment-resistant depression is comorbid with OSA. ${ }^{24}$ However, of the 115 depressive disorder patients who met the inclusion criteria in the present study, 51.3\% had OSA. Additionally, in the logistic regression analysis, the results showed a higher significant relation between AHI and diagnosis (MDDs or bipolar disorder [in a major depressive episode]), BMI, HAMD, and PSQI. Therefore, we may consider that there were many undiagnosed OSA cases in depression patients.

Of the 115 patients in the present study, 41 were diagnosed with bipolar depression and 74 were diagnosed with unipolar depression. Of the 59 depressive disorder patients

Table I Demographic data and clinical factors of the patients

\begin{tabular}{llll}
\hline Group & All patients & $\begin{array}{l}\text { OSA } \\
(\mathbf{A H I} \geq \mathbf{5})\end{array}$ & $\begin{array}{l}\text { Non-OSA } \\
(\mathbf{A H I}<\mathbf{5})\end{array}$ \\
\hline Subjects (n) & 115 & 59 & 56 \\
Sex, male/female (n) & $4 I / 74$ & $17 / 42$ & $24 / 32$ \\
Age (years), mean (SD) & $51.58(\mathrm{II} .48)$ & $53.42(12.8)$ & $48.86(10.55)$ \\
Diagnosis, MDD or BD (n) & $74 / 4 I$ & $43 / 16$ & $31 / 25$ \\
BMI (kg/m²), mean (SD) & $22.48(3.42)$ & $23.74(3.37)$ & $21.15(2.95)$ \\
HAMD, mean (SD) & $20.97(4.47)$ & $21.95(4.88)$ & $19.93(3.76)$ \\
HAMA, mean (SD) & $18.91(5.01)$ & $18.92(6.07)$ & $18.91(3.62)$ \\
PSQI, mean (SD) & $10.7(3.63)$ & $12.19(3.03)$ & $9.09(3.56)$ \\
\hline
\end{tabular}

Abbreviations: $\mathrm{AHI}$, apnea-hypopnea index; $\mathrm{BD}$, bipolar disorder; $\mathrm{BMI}$, body mass index; HAMD, Hamilton Rating Scale for Depression; HAMA, Hamilton Anxiety Rating Scale; MDD, major depressive disorder; OSA, obstructive sleep apnea; PSQI, Pittsburgh Sleep Quality Index; SD, standard deviation.
Table 2 Logistic regression of $\mathrm{AHI}$ and risk factors

\begin{tabular}{llllll}
\hline Risk factors & $\boldsymbol{\beta}$ & Wald & P-value & OR & $\mathbf{9 5 \%} \mathbf{C l}$ \\
\hline Diagnosis (MDD or BD) & 1.01 & 4.05 & 0.044 & 2.72 & $1.03-7.21$ \\
BMI & 0.32 & 13.3 & 0.000 & 1.38 & $1.16-1.65$ \\
PSQI & 0.24 & 12.1 & 0.001 & 1.28 & $1.11-1.46$ \\
HAMD & 0.14 & 5.05 & 0.02 & 1.15 & $1.02-1.29$ \\
\hline
\end{tabular}

Abbreviations: $\mathrm{AHI}$, apnea-hypopnea index; $\mathrm{BD}$, bipolar disorder; $\mathrm{BMI}$, body mass index; $\mathrm{Cl}$, confidence interval; HAMD, Hamilton Rating Scale for Depression; MDD, major depressive disorder; OR, odds ratio; PSQI, Pittsburgh Sleep Quality Index.

who met the OSA criteria, 16 were diagnosed with bipolar depressive disorder and 43 were diagnosed with unipolar depression. Meanwhile, the logistic test results showed that the risk of comorbid OSA in unipolar depression is 2.72 times that of bipolar depressive disorders. It is thought that the OSA diagnosis rate in MDD patients is higher than that in bipolar disorder patients, and the possibility cannot be ruled out that the sample included a high proportion of patients with MDD. Furthermore, a significant correlation was seen between the HAMD and PSQI scores and the AHI or OSA diagnosis. Thus, it seems that having a higher HAMD or PSQI score is associated with increased probability or possibility of having OSA. A significant correlation was seen between AHI and depressive severity, as well as sleep quality, according to HAMD and PSQI scores in the present patients.

Some studies have indicated that risk factors for OSA include sex, age, obesity, pharyngeal abnormalities, and cephalometric features..$^{20-22,25}$ In our results, significant associations were noted between the BMI and AHI or OSA diagnosis. It would be logical to assume that a higher BMI is associated with increased probability or possibility of having OSA. Therefore, it may be concluded that these risk factors (BMI, HAMD, PSQI, and diagnosis) would greatly increase the diagnosis rate of OSA in the psychiatric department. Depressed patients with the above risk factors or suspected OSA should be referred to a sleep disorder center for evaluation by PSG to confirm the diagnosis of OSA.

The limitations of our study design should also be noted. The symptom of daytime sleepiness was related to the severity of OSA. However, others found the predictive value of excessive daytime sleepiness for OSA to be low. ${ }^{21,25}$ In addition, Rosenthal and Dolan ${ }^{25}$ found that nonspecific daytime sleepiness may be more strongly observed in mood disorder patients than in the general population because of psychotropic drugs or the underlying disease itself. Clinical factors, such as cardiovascular disease, hypertension, and diabetes, have been shown to be associated with OSA. ${ }^{13}$ However, the sample size was relatively small in the present study. In the future, investigations with a larger sample 
should be conducted, to further investigate whether treatment for OSA might achieve further gains on mood.

\section{Conclusion}

Depressed patients may have potentially life-threatening OSA. Particular attention should be paid to depressive patients who are resistant to treatment, and psychiatrists should consider calling for voluntary OSA screening. The clinical risk factors (diagnosis [MDDs or bipolar disorder \{ in a major depressive episode $\}$ ], BMI, HAMD, and PSQI) could predict AHI or OSA diagnosis. The presence of these risk factors, including BMI, HAMD, diagnosis, and PSQI, is more efficient for recognizing OSA in mood disorders than a single risk factor. In clinical practice, physicians should not just treat the depression. It is important to screen patients at high risk of OSA when assessing patients for depression, especially those with unresponsive depression. Treatment of OSA could improve not only the compliance to pharmacological antidepressant treatment but also the treatment response rate for depression. ${ }^{14}$ Psychiatrists involved in treating mood disorders comorbid with OSA should be cautious in using drugs with a muscle relaxant effect such as benzodiazepines.

\section{Acknowledgments}

The study was supported by grants from the Science and Technology Development Plan Project of Hangzhou Municipality (number 20160533B57), the Medical and Health Science and Technology Plan Project of Hangzhou Municipality (number 2015A56), the Medical and Health Science and Technology Plan Project of Zhejiang Province (number 2017185411), and the Key Project of the Medical Platform of Zhejiang Province (number 2015ZDA016).

\section{Disclosure}

The authors report no conflicts of interest in this work.

\section{References}

1. American Psychiatric Association. Practice Guideline for the Treatment of Patients with Major Depressive Disorder. 3rd ed. Arlington County, VA: American Psychiatric Publishing Inc; 2010.

2. Arroll B, Macgillivray S, Ogston S, et al. Efficacy and tolerability of tricyclic antidepressants and SSRIs compared with placebo of or treatment of depression in primary care: a meta-analysis. Ann Fam Med. 2005;3:449-456.

3. Ruhe HG, Huyser J, Swinkels JA, Schene AH. Switching antidepressants after a first selective serotonin reuptake inhibitor in major depressive disorder: a systematic review. J Clin Psychiatry. 2006;67(12): $1836-1855$
4. Baghai TC, Moller HJ, Rupprecht R. Recent progress in pharmacological and non-pharmacological treatment options of major depression. Curr Pharm Des. 2006;12(4):503-515.

5. Souery D, Amsterdam J, Montigny C, et al. Treatment resistant depression: methodological overview and operational criteria. Eur Neuropsychopharmacol. 1999;9(1-2):83-91.

6. McIntyre RS, Filteau MJ, Martin L, et al. Treatment-resistant depression: definitions, review of the evidence, and algorithmic approach. J Affect Disord. 2014;156:1-7.

7. McMahon FJ, Buervenich S, Charney D, et al. Variation in the gene encoding the serotonin $2 \mathrm{~A}$ receptor is associated with outcome of antidepressant treatment. Am J Hum Genet. 2006;78(5):804-814.

8. Harris M, Glozier N, Ratnavadivel R, Grunstein RR. Obstructive sleep apnea and depression. Sleep Med Rev. 2009;13(6):437-444.

9. Riihimäki KA, Vuorilehto MS, Melartin TK, Isometsä ET. Five-year outcome of major depressive disorder in primary health care. Psychol Med. 2014;44(7):1369-1379.

10. Szaulińska K, Pływaczewski R, Sikorska O, et al. Obstructive sleep apnea in severe mental disorders. Psychiatr Pol. 2015;49(5):883-895.

11. Young T, Peppard PE, Gottlieb DJ. Epidemiology of obstructive sleep apnea: a population health perspective. Am J Respir Crit Care Med. 2002;165(9):1217-1239.

12. Farney RJ, Lugo A, Jensen RL, Walker JM, Cloward TV. Simultaneous use of antidepressant and antihypertensive medications increases likelihood of diagnosis of obstructive sleep apnea syndrome. Chest. 2004;125(4):1279-1285.

13. Schröder CM, O'Hara R. Depression and obstructive sleep apnea (OSA). Ann Gen Psychiatry. 2005;27:4-13.

14. Waterman L, Begley AE, Buysse DJ, Lenze E, Blumberger DM. Obstructive sleep apnea and antidepressant treatment response in older adults. Am J Geriatr Psychiatry. 2015;23(3):S97-S98.

15. Kerner N, Malovichko A, Pelton G, et al. Obstructive sleep apnea and risk of mild cognitive impairment in older adults with depression. $\mathrm{Am}$ J Geriatr Psychiatry. 2015;23(3):S110-S112.

16. Sanchez AI, Buela-Casal G, Bermudez MP, Casas-Maldonado F. The effects of continuous positive air pressure treatment on anxiety and depression levels in apnea patients. Psychiatry Clin Neurosci. 2001;55(6):641-646.

17. Means MK, Lichstein KL, Edinger JD, et al. Changes in depressive symptoms after continuous positive airway pressure treatment for obstructive sleep apnea. Sleep Breath. 2003;7(1):31-42.

18. Schwartz DJ, Kohler WC, Karatinos G. Symptoms of depression in individuals with obstructive sleep apnea may be amenable to treatment with continuous positive airway pressure. Chest. 2005;128(3):1304-1309.

19. Kawahara S, Akashiba T, Akahoshi T, Horie T. Nasal CPAP improves the quality of life and lessens the depressive symptoms in patients with obstructive sleep apnea syndrome. Intern Med. 2005;44(5):422-427.

20. Dixon JB, Schachter LM, O'Brien PE. Predicting sleep apnea and excessive day sleepiness in the severely obese: indicators for polysomnography. Chest. 2003;123(4):1134-1141.

21. Kubota Y, Nakayama H, Takada T, et al. Facial axis angle as a risk factor for obstructive sleep apnea. Intern Med. 2005;44(8):805-810.

22. Rodsutti J, Hensley M, Thakkinstian A, D'Este C, Attia J. A clinical decision rule to prioritize polysomnography in patients with suspected sleep apnea. Sleep. 2004;27(4):694-699.

23. Hattori M, Kitajima T, Mekata T, et al. Risk factors for obstructive sleep apnea syndrome screening in mood disorder patients. Psychiatry Clin Neurosci. 2009;63(3):385-391.

24. Lee S, Wing YK, Chen CN. Obstructive sleep apnoea and depression. Aust N Z J Psychiatry. 1993;27:165-166.

25. Rosenthal LD, Dolan DC. The Epworth sleepiness scale in the identification of obstructive sleep apnea. J Nerv Ment Dis. 2008; 196(5):429-431. 


\section{Publish your work in this journal}

Neuropsychiatric Disease and Treatment is an international, peerreviewed journal of clinical therapeutics and pharmacology focusing on concise rapid reporting of clinical or pre-clinical studies on a range of neuropsychiatric and neurological disorders. This journal is indexed on PubMed Central, the 'PsycINFO' database and CAS, and is the official journal of The International Neuropsychiatric Association (INA). The manuscript management system is completely online and includes a very quick and fair peer-review system, which is all easy to use. Visit http://www.dovepress.com/testimonials.php to read real quotes from published authors.

\footnotetext{
Submit your manuscript here: http://www.dovepress.com/neuropsychiatric-disease-and-treatment-journal
} 